\title{
How to Use the HAMNP Books
}

In essence, from data computerization point of view, scientific knowledge is the expression of interrelation between research objects in different types. During a long coastline without computer, people learn and spread scientific knowledge in traditional ways, including education, reading, and exchanging information with each other. In today's world, using computer's powerful functions, we have a new way to learn systematical, complete knowledge. In short, a study process in the new way is to search and learn some relationships. Next, we discuss concretely how to use the HAMNP books.

In these books, there are three kinds of data and three pairs of important relations. Three kinds of data are: (1) marine living sources (source); (2) secondary metabolites (compounds); and (3) pharmacological activities (pharm-activity). The three pairs of important relations are: (1) relationship between source and compounds; (2) relationship between compounds and pharm-activity; and (3) relationship between source and pharm-activity. In the case of asking questions, each relation has two directions; hence, together there are six types of questions:

Type 1: from known source to unknown compound

Type 2: from known compound to unknown source

Type 3: from known compound to unknown pharm-activity

Type 4: from known pharm-activity to unknown compound

Type 5: from known source to unknown pharm-activity

Type 6: from known pharm-activity to unknown source (Figure 1)

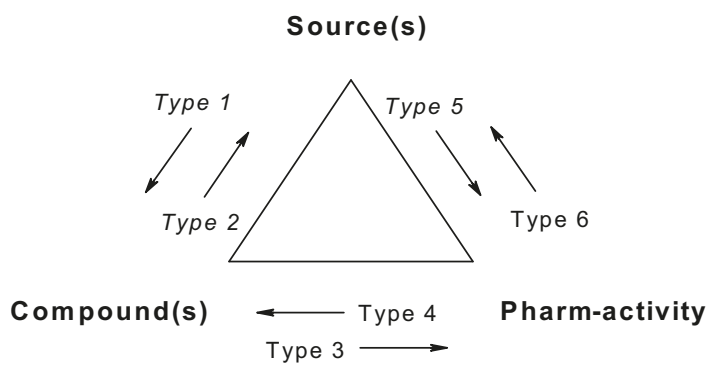

Figure 1: Kinds of Data and Six Types of Questions.

\section{(1) An Illustration of Type 1 (and Type 3, Type 5) Question}

Up to now, what alkaloids in volume 3 are isolated from sponges of genus Agelas? From index 3 of volume 3, one will get the following related data in detail:

Agelas cf. nemoechinata 507.

Agelas cf. mauritiana 449, 454, 471. 
Agelas clathrodes 344, 460, 461, 464, 468, 474, 478, 479, 480, 481, 483, 506, 506, 507, 509.

Agelas conifera 344, 449, 454, 468, 469, 471, 474, 478, 479, 480, 481, 506, 507, 509.

Agelas dendromorpha 446.

Agelas dispar 462, 463, 478, 479, 480, 481, 485, 506, 509, 1124.

Agelas flabelliformis 344.

Agelas linnaei 335, 336, 337, 338.

Agelas longissima 478, 479, 480, 481, 506, 506, 509.

Agelas mauritiana 345, 468, 487, 488, 506, 507.

Agelas nakamurai 445, 449, 469, 481, 504, 505, 509, 509, 510, 511, 512.

Agelas novaecaledoniae 449, 509.

Agelas oroides 238, 239, 344, 345, 506, 519.

Agelas sceptrum 506, 509.

Agelas schmidti 509.

Agelas sp. 64, 470, 485, 487, 490, 491, 500, 501, 502, 503.

Agelas sp. SS-1003 449, 454, 471, 477, 488, 492, 493, 494, 495, 496, 497, 498, 499, 506, 517, 519.

Agelas sventres 516.

Agelas wiedenmayeri 506.

Since all of compounds 1-1162 in volume 3 are alkaloids, the following 57 compounds $(64,238,239,335,336,337,338,344,345$, 446, 449, 454, 460-464, 468-471, 474, 477-481, 483, 485, 487, 488, 490-507, 509-512, 516, 517, 519, and 1124) are answers to the current question.

Then, readers can enjoy studying these 57 compounds by reading the book, including their pharm-activity (question of types 3 and 5). For example, with entry 506 (E)-Oroidin, a reader will know that the compound had already been isolated from following sponges in genus Agelas:

Agelas conifera (Caribbean, yield $=2.1 \% \mathrm{dw}$ ),

Agelas dispar (Caribbean, yield $=4.2 \% \mathrm{dw}$ ),

Agelas clathrodes (Caribbean, yield $=2.1 \% \mathrm{dw}$ ),

Agelas longissima (Caribbean, yield $=4.1 \% \mathrm{dw}$ ),

Agelas sp. SS-1003 (off Seragaki, Okinawa),

Agelas oroides,

Agelas conifera,

Agelas longissima,

Agelas mauritiana,

Agelas clathrodes,

Agelas wiedenmayeri,

Agelas sceptrum,

and from other sponges Axinella verrucosa, 
Axinella damicornis,

Hymeniacidon sp.,

Pseudaxinyssa cantharella,

Acanthella carteri and Acanthella aurantiaca.

And $(E)$-Oroidin has the following pharmacological activities:

Antibacterial (Micrococcus luteus, MIC $=4.07 \mu \mathrm{g} / \mathrm{mL}$;

Bacillus subtilis, $\mathrm{MIC}=8.33 \mu \mathrm{g} / \mathrm{mL}$;

Escherichia coli, $\mathrm{MIC}=33.3 \mu \mathrm{g} / \mathrm{mL}$ );

antibacterial (Staphylococcus aureus ATCC 25923, $\mathrm{IC}_{50}=0.96 \mu \mathrm{mol} / \mathrm{L}$,

Staphylococcus aureus ATCC 9144, $\mathrm{IC}_{50}=1.2 \mu \mathrm{mol} / \mathrm{L}$,

Bacillus subtilis ATCC 6051, $\mathrm{IC}_{50}=2.0 \mu \mathrm{mol} / \mathrm{L}$,

Bacillus subtilis ATCC 6633, $\mathrm{IC}_{50}=0.62 \mu \mathrm{mol} / \mathrm{L}$,

Escherichia coli ATCC 11775, $\mathrm{IC}_{50}=0.55 \mu \mathrm{mol} / \mathrm{L}$,

Pseudomonas aeruginosa ATCC 10145, $\left.\mathrm{IC}_{50}=1.4 \mu \mathrm{mol} / \mathrm{L}\right)$;

antifungal (Candida albicans ATCC 90028, $\mathrm{IC}_{50}=6.3 \mu \mathrm{mol} / \mathrm{L}$ );

protein phosphatase $2 \mathrm{~A}$ inhibitor $\left(\mathrm{IC}_{50}=50 \mu \mathrm{mol} / \mathrm{L}\right)$;

antibacterial (gram-positive and gram-negative bacteria, MIC $\approx 60 \mu \mathrm{g} / \mathrm{mL}$, moderate);

adrenergic antagonist;

serotonin antagonist;

antimuscarinic;

antifoulant;

antihistaminic (gpg ileum, apparent affinity of antagonistic effect $\mathrm{pD}_{2}=$ $4.02 \pm 0.11$, nonspecific noncompetitive effect);

IL-8 $\mathrm{R} \alpha$ receptor inhibitor $\left(\mathrm{IC}_{50}=9.6 \mu \mathrm{mol} / \mathrm{L}\right)$;

IL-8 R $\beta$ receptor inhibitor $\left(\mathrm{IC}_{50}=10.8 \mu \mathrm{mol} / \mathrm{L}\right)$;

protein kinase $\mathrm{C}$ inhibitor $\left(\mathrm{IC}_{50}=4.8 \mu \mathrm{mol} / \mathrm{L}\right)$;

antimalarial (Plasmodium falciparum K1 strain, $\mathrm{IC}_{50}=3.9-7.9 \mu \mathrm{g} / \mathrm{mL}$, MMOA:

FabI inhibition).<smiles>Nc1nc(/C=C/CNC(=O)c2cc(Br)c(Br)[nH]2)c[nH]1</smiles>

\section{(2) An Illustration of Type 4 (and Type 2, Type 6) Question}

"What are isolated alkaloids in volume 3 with pharmacological activity antitrypanosomal? And what are their marine sources ?” 
Table 2: Answer to the Above Type 4 Question.

\begin{tabular}{lll}
\hline $\begin{array}{l}\text { Vol. Code Compound } \\
\text { Name }\end{array}$ & Structure & $\begin{array}{l}\text { Related Sources } \\
\text { (Bass Strait, Tasmania, } \\
\text { Australia) }\end{array}$ \\
\end{tabular}

310 Didemnidine $\mathrm{A}$<smiles>C[NH2+]CCCC[NH2+]CCCNC(=O)C(=O)c1c[nH]c2ccccc12</smiles>

311 Didemnidine B

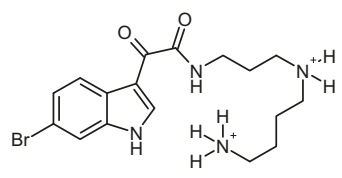

Ascidian Didemnum sp.

(Tiwai Pt, Southland, New Zealand)

\begin{tabular}{lll}
\hline$\ldots \ldots$ & $\ldots \ldots$ & $\ldots \ldots$ \\
\hline 3 & 936 & Zamamidine A
\end{tabular}

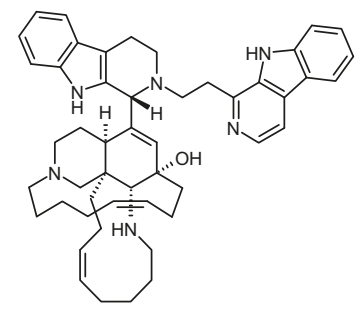

3938 Zamamidine C

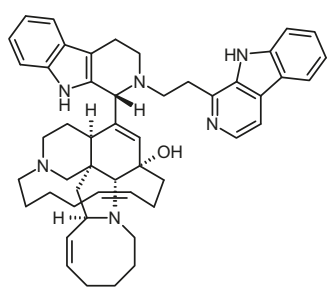

Ascidian Didemnum sp.

(Tiwai Pt, Southland, New Zealand)
Sponge Amphimedon sp. SS-975 (Seragaki, Okinawa)
Sponge Amphimedon sp. (Seragaki, Okinawa)

31089 Mariline $\mathrm{A}_{1}$

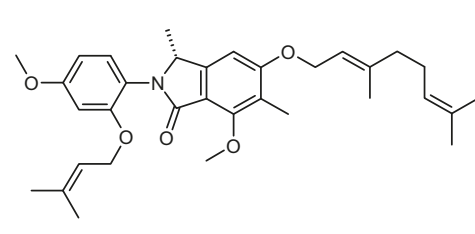

Marine-derived fungus Stachylidium sp. from sponge Callyspongia cf. flammea (location unspecified) 
To browse Index 5 of volume 3, searching “Antitrypanosomal,” the following results were obtained:

Antitrypanosomal, Trypanosoma brucei brucei 8, 93, 94, 901, 936, 938, 1089.

Antitrypanosomal, Trypanosoma brucei rhodesiense 10, 11, 876, 877.

Antitrypanosomal, Trypanosoma brucei selective 180, 181.

Antitrypanosomal, Trypanosoma brucei subsp. rhodesiense 852.

Further, from the entry bodies of the 14 compounds, all their sources can be obtained (see Table 2).

In summary, by using three parts of the books - the contents (ordered by structural classifications), the text (8,350 compound entries in volumes $1-8)$, and the indexes - readers can easily gain well-formatted systematically related knowledge in multidisciplinary fields. 
